\title{
The Functional Adaptation of the Diseased Kidney. I. Glomerular Filtration Rate *
}

\author{
Neal S. Bricker, $\dagger$ Saulo Klahr, and Richard E. Rieselbach $\vdots$ \\ (From the Renal Division, Department of Internal Medicine, Washington University School \\ of Medicine, St. Louis, Mo.)
}

In accounting for the functional capabilities as well as for certain of the functional limitations of the chronically diseased kidney, it has been postulated that, as the nephron population decreases, glomerular filtration rate (GFR) increases adaptively in the surviving nephrons $(1,2)$. This type of functional adaptation is reminiscent of the compensatory hypertrophy that occurs in a normal kidney after removal of the opposite organ (3, 4); however, support for its occurrence in the diseased kidney is largely circumstantial. Yet it is essential to the ultimate and complete understanding of the functional organization of the chronically diseased kidney to know whether or not this phenomenon actually occurs in advancing renal disease.

The basic difficulty in examining this question experimentally has been the lack of an appropriate experimental model. The detection of a change in the level of GFR per nephron requires not only the serial measurement of filtration rate, but either a direct method for counting the number of functioning nephrons, or an indirect, but meaning ful, index of the functioning nephron population. To count the functioning nephrons in the diseased kidney requires the quantitative correction for nonfiltering and other nonurine forming nephrons. Although the existence of these units may be detected in vivo in the experimentally diseased kidney (5), the available methods do not permit their accurate quantification. Thus the direct approach has not yet lent itself to a defini-

* Submitted for publication April 6, 1964; accepted June 17, 1964.

Supported by the National Institutes of Health (grant A-2667), the Department of the Army, Research and Development Branch (contract no. DA-49-007-MD-772), and a grant-in-aid from the American Heart Association.

† Work performed during the tenure of an established investigatorship, American Heart Association.

$\ddagger$ Research fellow, U. S. Public Health Service. tive analysis of the problem, although further refinements in methodology could make this possible.

In the present studies, the alternative approach has been taken. An indirect method has been used that provides a frame of reference for the functioning nephron population. This is less complex than the direct method, but it appears to bypass the methodologic barrier. The results provide unequivocal evidence that the experimentally diseased kidney of the dog is capable of undergoing a marked and sustained increase in GFR when the total (bilateral) nephron population is decreased.

\section{Methods}

One hundred and twenty-four experiments were performed on 26 female mongrel dogs. In each animal, two permanent hemibladders were constructed surgically to allow for the serial examination of renal function of both kidneys simultaneously. The experimental design was as follows: Control studies were performed when both kidneys were normal in order to establish the level of the GFR before there was any reduction in the nephron population. These measurements comprise the stage I studies. Pyelonephritis then was induced in one kidney of each animal, and the contralateral kidney was left unaltered. From 22 to 120 days later (mean 38 days) renal function was measured simultaneously in the diseased and contralateral control kidneys. These data comprise the stage II studies. In 5 dogs, two or more stage II studies were performed. The next step was designed to decrease the total number of functioning nephrons without decreasing further the nephron population of the diseased kidney. To accomplish this, the control kidney was either removed surgically (21 dogs) or subjected to ureteral ligation $(5 \operatorname{dog} s)$. The function of the diseased kidney then was restudied. The latter measurements, designated as stage III, were performed from 24 hours to 52 days after the removal or ureteral ligation of the control kidney. One study was performed in stage III on 15 dogs, and two or more studies were performed on 11 dogs. In each animal the values obtained from the diseased kidney in stage III were compared with the values from the same kidney in stage II. 
Experiments were performed either on hydropenic animals receiving mannitol infusions or on animals undergoing water diuresis. In each dog, the experimental conditions were identical in successive studies, except that the infusion rates were decreased in stage III to allow for the reduction in nephron population. GFR was measured in all experiments using the exogenous creatinine clearance, and creatinine was determined according to the method of Bonsnes and Taussky (6). In addition, the clearance of para-aminohippurate (PAH) was determined in 50 experiments on 14 animals. PAH was determined according to the method of Smith and associates (7).

The technique employed for the induction of experimental pyelonephritis was as follows: The animals were anesthetized with sodium pentobarbital, and one kidney was exposed through a flank incision. The renal parenchyma then was punctured approximately 400 times with an electrically heated 18 -gauge cautery needle. The punctures were applied diffusely over the entire renal parenchyma, and the depth of puncture was approximately $1.5 \mathrm{~cm}$, a distance sufficient to enter the medulla. In some animals, 50 or more additional punctures were made with a hypodermic needle repeatedly dipped into the broth culture of pathogenic bacteria. Electrocautery was not used in association with the latter needle tracts. One to $2 \mathrm{ml}$ of nutrient broth culture of Escherichia coli was administered intravenously through a peripheral vein. The organisms were obtained either from infected urine of patients or from dogs with pyelonephritis. They were isolated initially on MacConkey's medium and were incubated in plain nutrient broth for 4 to 6 hours before injection. The usual intravenous inoculum contained approximately $2 \times 10^{7}$ organisms. After completion of this procedure, and after assuring complete hemostasis, the kidney was replaced in the renal fossa and the wound closed.

The morphologic characteristics of the experimentally diseased kidneys are as follows: The involved organs become swollen and hyperemic during the first week following injection. Within 2 weeks, however, the renal parenchyma consistently demonstrates contraction and

TABLE I

Glomerular filtration rate (GFR) in dogs in which two or more studies were made in stage $I I^{*}$

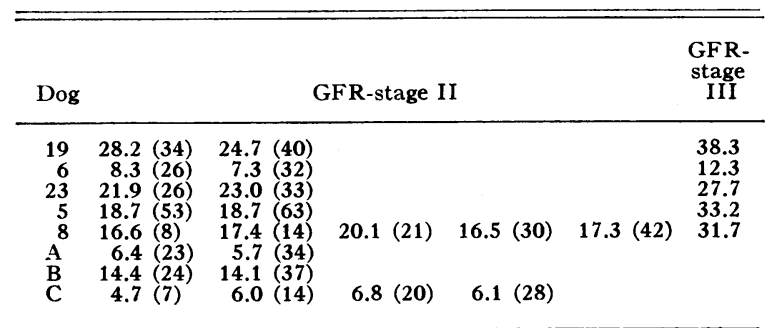

* Numbers designating dogs correspond to those used in Table II Numbers in parentheses indicate the interval in days following induction of experimental pyelonephritis. The three dogs labeled A, B, and C were not subjected to stage III studies. In two of these, disease was induced in the contralateral kidney; the third died after nephrectomy. scarring. Microscopically, the pathologic changes appear in radially oriented bands involving both cortex and medulla. Although the disease presumably originates in and around the needle tracts, the extension of the pathologic changes obscures the puncture sites and precludes establishment of the foregoing relationship. The microscopic abnormalities include mononuclear cell infiltration, tubular dilatation, intratubular casts, and fibrosis. Periodic acid Schiff stains demonstrate occasional thickening of glomerular basement membranes. The composite lesion resembles closely that seen in human chronic pyelonephritis. In most animals, viable bacteria may be recovered from the involved kidneys at autopsy, although the interval between induction and death may be many months.

GFR typically stabilizes within 2 weeks after induction of disease, and as long as the contralateral kidney remains free of disease, GFR rarely increases after the second to third week. The characteristic response is for values either to remain constant or to decrease with time. Serial studies on a representative animal with experimental pyelonephritis have been published previously (8), and data from several of the dogs in the present series in which more than one study was performed in stage II are shown in Table I.

\section{Results}

GFR in the diseased kidney increased in 25 of 26 dogs after eliminating the functional contribution of the contralateral control kidney. The average increment in GFR for the group between stages II and III was $60.6 \% .^{1}$ The average increment for the 21 animals subjected to unilateral nephrectomy was $58.1 \%$, whereas the value for the 5 animals subjected to ureteral ligation was $71 \%$. A complete tabulation of these data is included in Table II.

The results from the 11 dogs in which serial studies were performed in stage III are shown in Table III. A decrease in GFR with time would not be unexpected in view of the fact that the pyelonephritic lesions remain active as judged by both histologic and bacteriologic criteria; in addition the cystotomy tubes draining the hemibladders are prone to obstruction, and once this occurs renal function deteriorates rapidly. Despite these considerations, GFR tended either to remain constant or to increase with time. When all stage III values are averaged for each of the 11 dogs in which two or more studies were performed in stage III,

\footnotetext{
1 In the 11 dogs in which more than one study was performed in stage III, the maximal value was used for these calculations.
} 
TABLE II

Glomerular filtration rate in the experimentally diseased kidney of the dog before and after ureteral ligation or removal of the contralateral kidney

\begin{tabular}{|c|c|c|c|c|c|}
\hline \multirow[b]{3}{*}{ Dog } & \multirow{2}{*}{\multicolumn{2}{|c|}{ Stage II }} & \multicolumn{2}{|c|}{ Stage III } & \multirow[b]{3}{*}{$\begin{array}{l}\text { Per cent } \\
\text { increase }\end{array}$} \\
\hline & & & \multirow[b]{2}{*}{ GFR } & \multirow{2}{*}{$\begin{array}{l}\text { Days after } \\
\text { ureteral } \\
\text { ligation or } \\
\text { nephrectomy }\end{array}$} & \\
\hline & GFR & $\begin{array}{l}\text { Days after } \\
\text { induction }\end{array}$ & & & \\
\hline ' & $\mathrm{ml} / \mathrm{min}$ & & $m l / m i n$ & & \\
\hline \multicolumn{6}{|c|}{ Ureteral ligation } \\
\hline $\begin{array}{l}1 \\
2 \\
3 \\
4 \\
5\end{array}$ & $\begin{array}{r}3.4 \\
6.1 \\
10.2 \\
20.6 \\
18.7\end{array}$ & $\begin{array}{l}26 \\
45 \\
40 \\
26 \\
63\end{array}$ & $\begin{array}{r}6.9 \\
11.5 \\
16.8 \\
25.0 \\
33.2\end{array}$ & $\begin{array}{r}3 \\
2 \\
4 \\
6 \\
52\end{array}$ & $\begin{array}{r}102.9 \\
88.5 \\
64.7 \\
21.4 \\
77.5\end{array}$ \\
\hline Mean & 11.8 & & 18.7 & & 71.0 \\
\hline \multicolumn{6}{|c|}{ Nephrectomy } \\
\hline $\begin{array}{r}6 \\
7 \\
8 \\
9 \\
10 \\
11 \\
12 \\
13 \\
14 \\
15 \\
16 \\
17 \\
18 \\
19 \\
20 \\
21 \\
22 \\
23 \\
24 \\
25 \\
26\end{array}$ & $\begin{array}{r}7.3 \\
5.1 \\
17.3 \\
16.0 \\
11.0 \\
4.3 \\
14.4 \\
10.1 \\
10.4 \\
11.8 \\
13.7 \\
19.8 \\
22.9 \\
24.7 \\
5.0 \\
11.1 \\
25.5 \\
23.0 \\
13.0 \\
26.9 \\
18.2\end{array}$ & $\begin{array}{r}32 \\
120 \\
42 \\
31 \\
63 \\
27 \\
29 \\
26 \\
31 \\
27 \\
22 \\
27 \\
38 \\
34 \\
63 \\
32 \\
25 \\
33 \\
32 \\
27 \\
33\end{array}$ & $\begin{array}{r}12.3 \\
12.6 \\
31.7 \\
31.7 \\
17.5 \\
10.5 \\
16.6 \\
16.8 \\
14.7 \\
18.8 \\
24.3 \\
30.3 \\
43.3 \\
38.3 \\
6.1 \\
16.0 \\
28.4 \\
27.7 \\
20.4 \\
32.9 \\
15.9\end{array}$ & $\begin{array}{r}13 \\
28 \\
51 \\
12 \\
2 \\
11 \\
7 \\
13 \\
4 \\
13 \\
11 \\
11 \\
6 \\
14 \\
9 \\
5 \\
11 \\
19 \\
3 \\
22 \\
4\end{array}$ & $\begin{array}{r}68.5 \\
147.1 \\
83.2 \\
98.1 \\
59.1 \\
144.2 \\
15.3 \\
66.3 \\
41.3 \\
59.3 \\
77.4 \\
53.0 \\
89.1 \\
55.1 \\
22.0 \\
44.1 \\
11.4 \\
20.4 \\
56.9 \\
22.3 \\
-12.6\end{array}$ \\
\hline $\begin{array}{l}\text { Mean } \\
\text { value }\end{array}$ & 14.6 & 38 & 22.2 & & 58.1 \\
\hline \multicolumn{5}{|c|}{ Mean value for all dogs } & 60.6 \\
\hline
\end{tabular}

the mean of these averages is $53 \%$ greater than the corresponding stage II values.

Sequential measurements of $\mathrm{PAH}$ clearance were performed in 14 dogs (Table IV). In 5 of these, two studies and in a sixth dog four studies were performed in stage III. The average value obtained in stage III for all animals (using the maximal values in the 6 dogs with more than one study) was $66.5 \%$ greater than in stage II. ${ }^{2}$ The

\footnotetext{
2 The total increment in renal plasma flow per functioning nephron probably was greater than $66 \%$ in that $\mathrm{PAH}$ clearance increased in the normal kidneys between stages I and II by an average of $27.2 \%$. An equivalent increment may be presumed to have occurred in the residual nephrons of the diseased kidneys between stages I and II.
}

increase in GFR in the same animals for the same experiments was $69.8 \%$.

\section{Discussion}

GFR of the diseased kidney of the dog has been found to increase adaptively as the nephron population decreases. This was demonstrated by measuring GFR of the diseased organ first with a contralateral kidney contributing to function (stage II) and again after the function of the contralateral kidney had been eliminated (stage III). The total number of nephrons in the diseased organs could not have increased between stage II and stage III, unless nonfunctioning nephrons became 
TABLE III

Glomerular filtration rate in the 11 dogs in which two or more studies were made in stage $I I I^{*}$

\begin{tabular}{|c|c|c|c|c|c|}
\hline \multirow[t]{2}{*}{ Dog } & $\begin{array}{l}\text { GFR- } \\
\text { stage II }\end{array}$ & \multicolumn{4}{|c|}{ GFR-stage III } \\
\hline & $\mathrm{ml} / \mathrm{min}$ & & & & \\
\hline $\begin{array}{r}8 \\
14 \\
19 \\
11 \\
7 \\
13 \\
15 \\
16 \\
10 \\
9 \\
5\end{array}$ & $\begin{array}{r}17.3 \\
10.4 \\
24.7 \\
4.3 \\
5.1 \\
10.1 \\
11.8 \\
13.7 \\
11.0 \\
16.0 \\
18.7\end{array}$ & $\begin{aligned} & 22.9(21) \\
& 14.7(4)(4) \\
& 32.6(6) \\
& 8.7(5) \\
& 6.9(7) \\
& 16.8(13) \\
& 12.6(4) \\
& 14.5(2) \\
& 17.5(2) \\
& 27.1(3) \\
& 18.9(2)\end{aligned}$ & $\begin{aligned} 23.7(24) \\
13.6(21) \\
38.3(14) \\
10.5(8) \\
7.8(9) \\
16.4(41) \\
18.8(13) \\
24.3(11) \\
17.2(33) \\
31.7(12) \\
33.2(52)\end{aligned}$ & $\begin{array}{l}30.0(37) \\
12.9(31) \\
32.2(41) \\
10.5(11) \\
12.6(28)\end{array}$ & $\begin{array}{l}31.7(51) \\
13.1(37)\end{array}$ \\
\hline
\end{tabular}

* Numbers designating dogs correspond to those used in Table II. Numbers in parentheses indicate the number of days following ablation
of function of control kidney.

functional. Rather, it seems more likely that any change in nephron population would consist of a decrease, due to progression of the parenchymal disease. Hence, the increments in GFR that were observed in 25 out of $26 \mathrm{dogs}$, and which for the group averaged $60.6 \%$, suggest that the average value for GFR per functioning nephron increased adaptively.

The mechanisms initiating the increase in GFR are unknown. That hemodynamic factors resulting from rerouting of blood from the low pressure vascular bed of the control kidney may play a role is suggested by the marked increments in GFR observed as early as 24 hours after ablation of the function of the control kidney. On the other hand, this could not represent the sole explanation inasmuch as the magnitude of the in-

TABLE IV

Clearance of para-aminohippurate $(P A H)$ in the experimentally diseased kidney of the dog before and after ablation of function of the contralateral uninvolved kidney*

\begin{tabular}{|c|c|c|c|c|c|}
\hline \multicolumn{2}{|c|}{ Dog Stage II } & Stage III & & & \\
\hline & $m l / \min$ & $m l / \min$ & & & \\
\hline $\begin{array}{r}2 \\
1 \\
15 \\
16 \\
17 \\
19 \\
3 \\
18 \\
13 \\
14 \\
10 \\
24 \\
9 \\
8\end{array}$ & $\begin{array}{l}20.0 \\
9.0 \\
63.2 \\
46.6 \\
51.9 \\
75.4 \\
38.0 \\
87.2 \\
35.9 \\
28.6 \\
30.1 \\
42.0 \\
55.9 \\
69.1\end{array}$ & $\begin{aligned} 28.6(2) \\
23.4(3) \\
87.0(13) \\
85.9(11) \\
85.3(11) \\
103.5(14) \\
58.5(4) \\
133.0(6) \\
56.5(13) \\
44.9(11) \\
65.0(2) \\
64.2(3) \\
94.0(3) \\
81.3(21)\end{aligned}$ & $\begin{array}{r}54.6(41) \\
44.8(37) \\
60.6(33) \\
87.4(10) \\
102.8(12) \\
90.0(24)\end{array}$ & 108.0 & 124.3 \\
\hline
\end{tabular}

* Numbers designating dogs correspond to those used in Table II. Numbers in parentheses indicate the number of days following ablation of function of control kidney. crement was no less (and indeed it appeared to be somewhat greater) in those animals subjected to ureteral ligation than in those in which the control kidney was removed (Table II) ; yet in the former group, it may be assumed that an appreciable amount of blood continued to flow through the obstructed kidneys $(9,10)$. The data obtained from the animals with unilateral hydronephrosis also imply that the increment in GFR is largely independent of the number of perfused nephrons, in contradistinction to the number of urine-forming nephrons. The rapidity of onset of the adaptive increment speaks against the possibility that retention of some unidentified substance is primarily responsible for the adaptation. Whether hormonal factors influence these changes, as they appear to influence compensatory hypertrophy in a normal kidney $(11,12)$, cannot be evaluated at the present time. Detailed exploration of the mechanisms responsible for the increase in filtration rate in the diseased kidney may well provide a fertile field for future investigation.

From the point of view of the functional organization of a nephron, chronic hyperfiltration has intriguing implications. This single change, in a function that is mediated entirely by physical forces, must evoke major alterations in energydependent tubular transport systems. The most obvious effect of hyperfiltration would be to increase the excretory capacity of a nephron. However, if the increase in GFR is appreciable, it must alter profoundly the transport requirements of the tubular epithelial cells.

Changes in excretory patterns. Hyperfiltration will result in an increase in the filtered load of all filtered solutes, and unless $100 \%$ of the additional filtered load is reabsorbed, the excretion rate (all other things being equal) must increase. This is clearly evident for substances that are excreted predominantly by glomerular filtration. For example, for any given number of nephrons, an adaptive increment in GFR per nephron would increase the rate of excretion of creatinine and urea and would slow the rate of accumulation of these solutes in body fluids with advancing disease.

Hyperfiltration would contribute importantly to the maintenance of sodium balance on an unrestricted salt intake. For this balance to be maintained, the average daily rate of excretion of so- 
dium per nephron must increase as the number of surviving nephrons decreases. A progressive increment in the filtered load of sodium per nephron would greatly facilitate this regulation.

Hyperfiltration also would affect the excretion, and the maintenance of balance, of actively transported species other than sodium ions. For exexample, the excretion rate of phosphate relative to the filtered load increases as renal disease advances (13). One mechanism proposed for this adaptation is secondary hyperparathyroidism. However, hyperfiltration could contribute to the relative phosphaturia.

The implications of hyperfiltration might even extend to protein excretion. For example, in pyelonephritis, pathologic involvement of the glomeruli is unusual, yet proteinuria occurs with considerable frequency. If some protein normally enters the glomerular filtrate, an increase in GFR per nephron should lead to a concomitant increase in this physiologic protein filtration, and unless the tubular reabsorption of protein increased to equal the filtered load, protein would escape into the urine. ${ }^{3}$

Changes in tubular transport. There is a growing body of evidence to support the view that a considerable portion of the work of the renal tubular epithelial cells is involved in the active transport of sodium $(14,15)$. Simple calculations dictate that if the average value for GFR per nephron increases by $50 \%$, the great majority of the extra filtered sodium must be reabsorbed, even though the excretion rate (per nephron) increases by as much as tenfold. Thus in advanced renal disease, it is very unusual for more than 15 to $20 \%$ of the total filtered load of sodium to be excreted (16). It is implicit, therefore, that the increase in the filtered load far exceeds the increase in excretion. Hence, the rate of active sodium transport per nephron must be supernormal under basal conditions, and hyperfiltration will lead to an increase in work and an associated increase in energy production in each surviving nephron.

3 The hyperperfusion of glomerular capillaries that appears to accompany hyperfiltration (i.e., renal plasma flow as well as GFR increased adaptively) might also contribute to proteinuria by decreasing the barrier to protein filtration and thereby increasing further the filtered load of protein entering the tubular fluid.
Hyperfiltration would also necessitate an alteration in the rate limiting events controlling active transport of other species. For example, there would be an increase in the filtered load of glucose and amino acids per nephron, and complete conservation of these solutes would require an augmentation of transport rates under steady state conditions. Observations to be reported separately show an increase in the maximal velocity of transport of PAH (17) and ammonium (18) in uremic dogs. The latter substances, however, are both secreted, and the influence of filtration rate on the adaptive changes is not apparent.

The extrapolation of the results of these studies to man with naturally occurring chronic renal disease must be made with a certain degree of caution. In the latter situation, the rate of progression of the disease may be extremely slow, and the decrease in nephron population would occur gradually. In the present studies, the nephron population was decreased abruptly, although in those animals in which the ureter of the control kidney was ligated, the number of nephrons contributing to urine formation was decreased without a corresponding decrement in the number of viable nephrons, or in the blood supply to these nephrons. Nevertheless, it may be concluded from these studies that the severely diseased kidney is capable of undergoing a marked and sustained increase in GFR. Presumably the same adaptation could occur in chronic progressive renal disease in man. If so, the possibility exists that GFR may increase to different degrees in different diseases, and this phenomenon could well explain some of the functional differences among various forms of chronic Bright's disease. For example, in glomerulonephritis, hypertension, and other diseases that preferentially affect either the glomeruli or the preglomerular arterioles, the increment in GFR per nephron could be less marked than in pyelonephritis. This could contribute to the differences observed in group plots of patients with glomerulonephritis versus those with pyelonephritis $(19,20)$. Thus, with a greater GFR per nephron, one would expect a greater saltlosing tendency, and an earlier loss of concentrating ability. The fact that acidosis appears to occur somewhat earlier in pyelonephritis than in glomerulonephritis might also be more amenable 
to explanation if, for a given filtration rate, there were fewer nephrons in pyelonephritis than in glomerulonephritis.

One final point deserves emphasis. The present studies underscore the need to differentiate conceptually between solute diuresis due to hyperfiltration and that due to an osmotic diuresis such as might be induced by high filtered loads of urea in the uremic state. In the former situation, net transport of sodium and other solutes is increased, whereas in the latter, net reabsorption of sodium is diminished. The implications of sodium loss during salt deprivation could be quite different (in terms of the functional integrity of the nephron) if net sodium reabsorption were supernormal than if it were decreased.

\section{Summary}

These experiments were designed to investigate the capacity of the residual nephrons of the experimentally diseased kidney of the dog to undergo an adaptive increase in glomerular filtration. Glomerular filtration rate (GFR) was measured in both kidneys of 26 dogs with unilateral renal disease. Thereafter, the uninvolved contralateral kidney was either removed $(21 \mathrm{dogs})$ or its ureter ligated ( 5 dogs). GFR increased in the diseased kidneys in 25 of the 26 animals; the average increment for the group was $60.6 \%$. Renal plasma flow was measured in 14 of these animals, and the average increment in this function was $66.5 \%$. The mechanisms underlying the adaptive increase in filtration rate are poorly understood; however, the influence of this adaptation on the functional characteristics of the nephron is striking. Hyperfiltration of the degree noted in these studies must lead not only to an increase in excretory capacity but to an increase in active transport of a number of solutes including sodium. Supernormal rates of sodium transport under basal conditions in turn would necessitate an increase in basal energy production and utilization per nephron.

If similar degrees of hyperfiltration occur in man with chronic renal disease, this adaptation could contribute importantly both to the functional capabilities and to certain of the functional limitations observed in the chronically diseased kidney.

\section{References}

1. Platt, R. Structural and functional adaptation in renal failure. Brit. med. J. 1952, 1, 1313.

2. Bricker, N. S. Chronic progressive renal disease: pathologic physiology and relation to treatment. Progr. cardiovasc. Dis. 1961, 4, 170. Heart, Kidney and Electrolytes, C. K. Friedberg, Ed. New York, Grune \& Stratton, 1962.

3. Addis, T., B. A. Myers, and J. Oliver. The regulation of renal activity. IX. The effect of unilateral nephrectomy on the function and structure of the remaining kidney. Arch. intern. Med. 1924, 34, 243.

4. Kohlberg, A. Relations of renal tubular and glomerular function as influenced by 75 per cent reduction of nephron population. Scand. J. clin. Lab. Med. 1959, 11 (suppl.), 41.

5. Damadian, R. V., E. Shwayri, and N. S. Bricker. Demonstration of a population of nephrons in diseased kidneys with perfused glomeruli that do not contribute to urine formation. In preparation.

6. Bonsnes, R. W., and H. H. Taussky. On the colorimetric determination of creatinine by the Jaffe reaction. J. biol. Chem. 1945, 158, 581.

7. Smith, H. W., N. Finkelstein, L. Aliminosa, B. Crawford, and M. Graber. The renal clearances of substituted hippuric acid derivatives and other aromatic acids in dog and man. J. clin. Invest. 1945, 24, 388.

8. Bricker, N. S., P. A. F. Morrin, and S. W. Kime, Jr. The pathologic physiology of chronic Bright's disease: an exposition of the "intact nephron hypothesis.” Amer. J. Med. 1960, 28, 77.

9. Selkurt, E. E. Effect of ureteral blockage on renal blood flow and urinary concentrating ability. Amer. J. Physiol. 1963, 205, 286.

10. Levy, S. E., M. F. Mason, T. R. Harrison, and A. Blalock. The effects of ureteral occlusion on the blood flow and oxygen consumption of the kidneys of unanesthetized dogs. Surgery 1937, 1, 238.

11. Winternitz, M. C., and L. L. Waters. The effect of hypophysectomy on compensatory renal hypertrophy in dogs. Yale J. Biol. Med. 1940, 12, 705.

12. Zeckwer, I. T. Compensatory growth of the kidney after unilateral nephrectomy in thyroidectomized rats. Amer. J. Physiol. 1946, 145, 681.

13. Goldman, R., and S. H. Bassett. Phosphorous excretion in renal failure. J. clin. Invest. 1954, 33, 1623.

14. Kramer, J., and P. Deetjan. Sodium reabsorption and oxygen consumption in the mammalian kidney. Proc. 1st Int. Congr. Nephrol. 1961, 687.

15. Thaysen, J. H., N. A. Lassen, and O. Munck. Sodium transport and oxygen consumption in the mammalian kidney. Proc. 1st Int. Congr. Nephrol. 1961, 692. 
16. Nickel, J. F., P. B. Lowrance, E. Leifer, and S. E. Bradley. Renal function, electrolyte excretion and body fluids in patients with chronic renal insuffciency before and after sodium deprivation. $\mathrm{J}$. clin. Invest. 1953, 32, 68.

17. Rieselbach, R. E., L. E. Todd, M. Rosenthal, and N. S. Bricker. Adaptive capacity of the residual nephrons of the chronically diseased kidney in the dog (abstract). J. Lab. clin. Med. 1963, 62, 1008.

18. Dorhout Mees, E. J., M. Machado, and N. S. Bricker. Unpublished observations.
19. Hodler, J. Etude de l'acidose au cours de pyélonéphrites, de glomerulonéphrites et de nephroscleroses avancées. Proc. 1st Int. Congr. Nephrol. 1961, 611.

20. Brod, J., V. Prat, and R. Dejdar. Early functional diagnosis of chronic pyelonephritis with some remarks on the pathogenesis of the pyelonephritic contracted kidney in Biology of Pyelonephritis, Henry Ford International Symposium. Boston, Little, Brown, 1960, p. 311. 